\title{
Braids, complex volume and cluster algebras
}

\author{
KAZUHIRO HIKAMI \\ REI INOUE
}

\begin{abstract}
We try to give a cluster-algebraic interpretation of the complex volume of knots. We construct the R-operator from cluster mutations, and show that it can be regarded as a hyperbolic octahedron. The cluster variables are interpreted as the edge parameters used by Zickert for computing complex volume.
\end{abstract}

$57 \mathrm{M} 25 ; 13 \mathrm{~F} 60$

\section{Introduction}

Interest in geometrical properties of quantum invariants has risen since the formulation of the volume conjecture (see Kashaev [17] and H Murakami and J Murakami [20]), which suggests a relationship between the colored Jones polynomial and the hyperbolic volume of knot complements. As quantum invariants of knots, such as the colored Jones polynomial, are constructed by use of the Artin braid relation, it is interesting to study a hyperbolic-geometric solution to the braid relation.

A fundamental object in 3-dimensional hyperbolic geometry is the ideal tetrahedron. When a manifold is constructed from a set of ideal tetrahedra, its complex volume, ie a complexification of hyperbolic volume, is written in terms of the extended Rogers dilogarithm function; see Neumann [23]. On the other hand, cluster algebras have been developed since the pioneering work of Fomin and Zelevinsky [9], and have applications in representation theory, Teichmüller theory, integrable systems and so on. The dilogarithm function also plays an important role in the theory of cluster algebras; see Fock and Goncharov [7] and Nakanishi [22].

The purpose of this article is to give a cluster-algebraic interpretation of the complex volume of knots. In our previous paper [13], we gave an interpretation of the cluster mutation as a hyperbolic ideal tetrahedron, and we proposed a method to compute the complex volume of 2-bridge knots. In this article, we first give a geometric interpretation of the $\mathrm{R}$-operator, which can be constructed from the cluster mutation based on a relation with Teichmüller theory; see Dynnikov [5] and Dehornoy, Dynnikov, Rolfsen and Wiest [4]. We find that the R-operator in Theorem 2.3 is identified with a 
hyperbolic octahedron composed of four ideal tetrahedra, and that the cluster variable corresponds to an edge parameter used by Zickert [29] to computate complex volume. Our main claims are in Theorem 3.1 and Conjecture 3.2: following a method of Zickert, we propose a formula for complex volume in terms of cluster variables. Our construction can be naturally quantized with the help of the quantum cluster algebra [12].

This paper is organized as follows. In Section 2, after explaining the minimal basics of the cluster algebra, we introduce the R-operator. In Section 3, we interpret the R-operator in hyperbolic geometry, and formulate the complex volume of knots in Theorem 3.1 and Conjecture 3.2. Some examples of numerical calculations are presented in Section 4.

\section{Cluster algebra and braid relation}

\subsection{Cluster variable}

We briefly introduce a notion of cluster algebra used in this article. A basic reference is [9].

A cluster seed $(\boldsymbol{x}, \boldsymbol{B})$ is a pair consisting of:

- A cluster variable $\boldsymbol{x}=\left(x_{1}, \ldots, x_{N}\right)$ (an $N$-tuple of algebraically independent variables).

- An exchange matrix $\boldsymbol{B}=\left(b_{i j}\right)$ (an $N \times N$ skew symmetric integer matrix).

For each $k=1, \ldots, N$, we define the mutation $\mu_{k}$ of $(\boldsymbol{x}, \boldsymbol{B})$ by

$$
\mu_{k}(\boldsymbol{x}, \boldsymbol{B})=(\tilde{\boldsymbol{x}}, \widetilde{\boldsymbol{B}}),
$$

where

- $\tilde{\boldsymbol{x}}=\left(\tilde{x}_{1}, \ldots, \tilde{x}_{N}\right)$ is

$$
\tilde{x}_{i}= \begin{cases}x_{i} & \text { for } i \neq k, \\ \frac{1}{x_{k}}\left(\prod_{j: b_{j k}>0} x_{j}^{b_{j k}}+\prod_{j: b_{j k}<0} x_{j}^{-b_{j k}}\right) & \text { for } i=k,\end{cases}
$$

- $\tilde{\boldsymbol{B}}=\left(\tilde{b}_{i j}\right)$ is

$$
\tilde{b}_{i j}= \begin{cases}-b_{i j} & \text { for } i=k \text { or } j=k, \\ b_{i j}+\frac{\left|b_{i k}\right| b_{k j}+b_{i k}\left|b_{k j}\right|}{2} & \text { otherwise. }\end{cases}
$$

The pair $(\tilde{\boldsymbol{x}}, \widetilde{\boldsymbol{B}})$ is again a cluster seed. We remark that the mutation $\mu_{k}$ is involutive, and that we have $\mu_{j} \mu_{k}(\boldsymbol{x}, \boldsymbol{B})=\mu_{k} \mu_{j}(\boldsymbol{x}, \boldsymbol{B})$ if $b_{j k}=0$. 
In terms of the cluster variable $\boldsymbol{x}$, we introduce the $y$-variable $\boldsymbol{y}=\left(y_{1}, \ldots, y_{N}\right)$ defined by Fomin and Zelevinsky [10]:

$$
y_{j}=\prod_{k} x_{k}^{b_{k j}} .
$$

The mutation $\mu_{k}$ induces a mutation of a pair $(\boldsymbol{y}, \boldsymbol{B})$,

$$
\mu_{k}(\boldsymbol{y}, \boldsymbol{B})=(\tilde{\boldsymbol{y}}, \tilde{\boldsymbol{B}})
$$

where $\tilde{\boldsymbol{B}}$ is $(2-3)$, and $\tilde{\boldsymbol{y}}=\left(\tilde{y}_{1}, \ldots, \tilde{y}_{N}\right)$ with $\tilde{y}_{j}=\prod_{k} \tilde{x}_{k}^{\tilde{b}_{k j}}$ given by

$$
\tilde{y}_{i}= \begin{cases}y_{k}^{-1} & \text { for } i=k, \\ y_{i}\left(1+y_{k}^{-1}\right)^{-b_{k i}} & \text { for } i \neq k, b_{k i} \geq 0, \\ y_{i}\left(1+y_{k}\right)^{-b_{k i}} & \text { for } i \neq k, b_{k i} \leq 0 .\end{cases}
$$

\subsection{R-operator}

We define the 7 by 7 exchange matrix $\boldsymbol{B}$ by

$$
\boldsymbol{B}=\left(\begin{array}{rrrrrrr}
0 & 1 & -1 & 0 & 0 & 0 & 0 \\
-1 & 0 & 0 & 1 & 0 & 0 & 0 \\
1 & 0 & 0 & -1 & 0 & 0 & 0 \\
0 & -1 & 1 & 0 & 1 & -1 & 0 \\
0 & 0 & 0 & -1 & 0 & 0 & 1 \\
0 & 0 & 0 & 1 & 0 & 0 & -1 \\
0 & 0 & 0 & 0 & -1 & 1 & 0
\end{array}\right)
$$

By regarding the matrix element as

$$
b_{i j}=\#\{\text { arrows from } i \text { to } j\}-\#\{\text { arrows from } j \text { to } i\},
$$

the exchange matrix $\boldsymbol{B}$ corresponds to quiver, which is dual to triangulated surface (see Fomin, Shapiro and D Thurston [8]). In our case (2-7), we have the quiver and the triangulated disk depicted in Figure 1.

For our later use, we introduce the R-operator acting on the cluster variables associated with the quiver in Figure 1.

Definition 2.1 We define the R-operator by

$$
\mathrm{R}=s_{3,5} s_{2,5} s_{3,6} \mu_{4} \mu_{2} \mu_{6} \mu_{4} \text {. }
$$



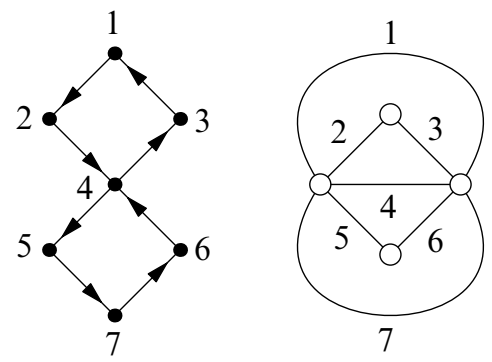

Figure 1: Quiver and triangulated disk

Here we have used the permutation $s_{i, j}$ of subscripts $i$ and $j$ in seeds,

$$
s_{i, j}\left(\ldots, x_{i}, \ldots, x_{j}, \ldots\right)=\left(\ldots, x_{j}, \ldots, x_{i}, \ldots\right) .
$$

Actions on the exchange matrix are defined in the same manner. Note that we have

$$
\mathrm{R}^{-1}=s_{3,6} s_{2,5} s_{3,5} \mu_{4} \mu_{5} \mu_{3} \mu_{4} .
$$

The permutations are included in the R-operator so that the exchange matrix $\boldsymbol{B}$ (2-7) is invariant under R. Explicitly we have

$$
\mathrm{R}^{ \pm 1}(\boldsymbol{x}, \boldsymbol{B})=\left(\mathrm{R}^{ \pm 1}(\boldsymbol{x}), \boldsymbol{B}\right),
$$

where

$$
\begin{aligned}
& \mathrm{R}(\boldsymbol{x})=\left(\begin{array}{c}
x_{1} \\
x_{5} \\
\frac{x_{1} x_{3} x_{4} x_{5}+x_{3} x_{4}^{2} x_{5}+x_{1} x_{3} x_{5} x_{7}+x_{3} x_{4} x_{5} x_{7}+x_{1} x_{2} x_{6} x_{7}}{x_{2} x_{4} x_{6}} \\
\frac{x_{3} x_{4} x_{5}+x_{3} x_{5} x_{7}+x_{2} x_{6} x_{7}}{x_{4} x_{6}} \\
x_{3} \\
x_{7}
\end{array}\right)^{\top}, \\
& \mathrm{R}^{-1}(\boldsymbol{x})=\left(\begin{array}{c}
x_{1} \\
\frac{x_{1} x_{3} x_{5}+x_{1} x_{2} x_{6}+x_{2} x_{4} x_{6}}{x_{3} x_{4}} \\
x_{6} \\
\frac{x_{1} x_{2} x_{4} x_{6}+x_{2} x_{4}^{2} x_{6}+x_{1} x_{3} x_{5} x_{7}+x_{1} x_{2} x_{6} x_{7}+x_{2} x_{4} x_{6} x_{7}}{x_{3} x_{4} x_{5}} \\
x_{2} \\
\frac{x_{2} x_{4} x_{6}+x_{3} x_{5} x_{7}+x_{2} x_{6} x_{7}}{x_{4} x_{5}} \\
x_{7}
\end{array}\right)^{\top} .
\end{aligned}
$$


Correspondingly, actions of the R-operator, (2-9) and (2-10), on the $y$-variable are respectively given as follows:

$$
\begin{aligned}
& \mathrm{R}(\boldsymbol{y})=\left(\begin{array}{c}
y_{1}\left(1+y_{2}+y_{2} y_{4}\right) \\
\frac{y_{2} y_{4} y_{5} y_{6}}{1+y_{2}+y_{6}+y_{2} y_{6}+y_{2} y_{4} y_{6}} \\
\frac{1+y_{2}+y_{6}+y_{2} y_{6}+y_{2} y_{4} y_{6}}{y_{2} y_{4}} \\
\frac{y_{4}}{\left(1+y_{2}+y_{2} y_{4}\right)\left(1+y_{6}+y_{4} y_{6}\right)} \\
\frac{1+y_{2}+y_{6}+y_{2} y_{6}+y_{2} y_{4} y_{6}}{y_{4} y_{6}} \\
\frac{y_{2} y_{3} y_{4} y_{6}}{1+y_{2}+y_{6}+y_{2} y_{6}+y_{2} y_{4} y_{6}} \\
\left(1+y_{6}+y_{4} y_{6}\right) y_{7}
\end{array}\right)^{\top} \\
& \mathrm{R}^{-1}(\boldsymbol{y})=\left(\begin{array}{c}
\frac{y_{1} y_{3} y_{4}}{1+y_{4}+y_{3} y_{4}} \\
\frac{y_{5}}{1+y_{4}+y_{3} y_{4}+y_{4} y_{5}+y_{3} y_{4} y_{5}} \\
\left(1+y_{4}+y_{3} y_{4}+y_{4} y_{5}+y_{3} y_{4} y_{5}\right) y_{6} \\
\frac{\left(1+y_{4}+y_{3} y_{4}\right)\left(1+y_{4}+y_{4} y_{5}\right)}{y_{3} y_{4} y_{5}} \\
y_{2}\left(1+y_{4}+y_{3} y_{4}+y_{4} y_{5}+y_{3} y_{4} y_{5}\right) \\
\frac{y_{3}}{1+y_{4}+y_{3} y_{4}+y_{4} y_{5}+y_{3} y_{4} y_{5}} \\
\frac{y_{4} y_{5} y_{7}}{1+y_{4}+y_{4} y_{5}}
\end{array}\right)^{\top}
\end{aligned}
$$

It should be remarked that the $\mathrm{R}-$ operator (2-9) can be also written as

$$
\mathrm{R}=s_{2,5} s_{3,6} \mu_{2} \mu_{6} \mu_{4} \mu_{2} \mu_{6},
$$

which can be checked from

$$
\begin{aligned}
& s_{3,5}\left(\mu_{3} \mu_{5} \mu_{4}\right)^{3}=1, \\
& s_{2,6}\left(\mu_{2} \mu_{6} \mu_{4}\right)^{3}=1 .
\end{aligned}
$$

These identities correspond to a (half) periodicity in the cluster algebra associated to $A_{3}$-type quiver, which is a subquiver of Figure 1. See [9;22].

\subsection{Braid relation}

We generalize the quiver in Figure 1 to that in Figure 2. Therein also given is the triangulated disk, and an exchange matrix $\boldsymbol{B}$ is given by the rule (2-8) as a generalization of (2-7). 


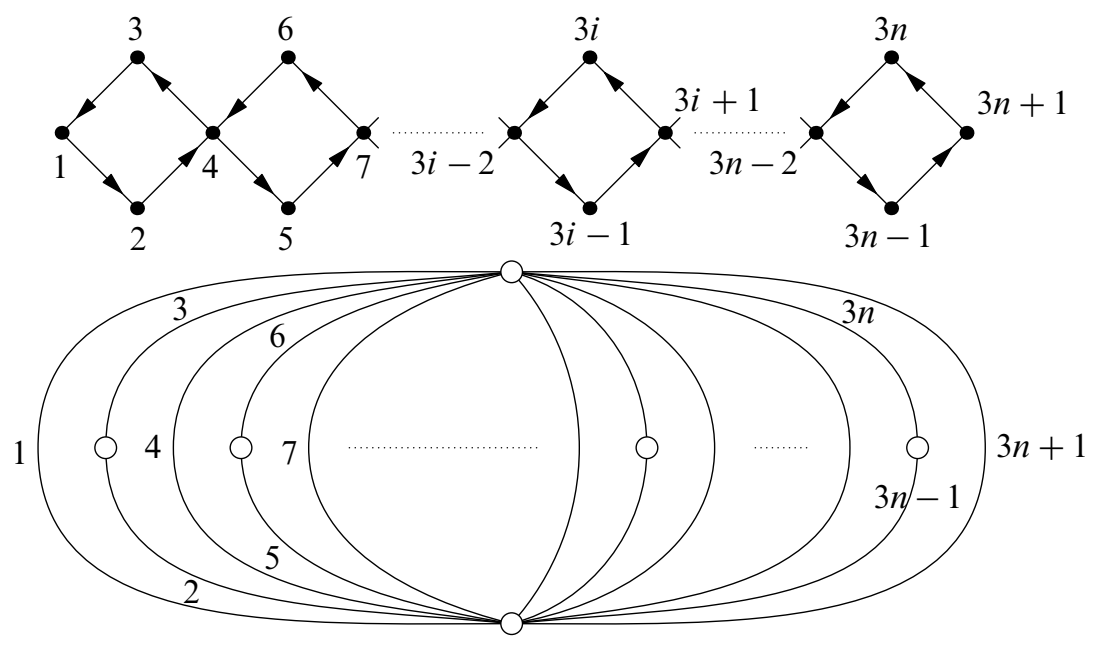

Figure 2: Quiver and triangulated disk

Definition 2.2 As a generalization of (2-9), we define the $\mathrm{R}$-operator $\stackrel{i}{\mathrm{R}}$ for $i=$ $1, \ldots, n-1$ associated with the quiver in Figure 2 by

$$
\stackrel{i}{\mathrm{R}}=s_{3 i, 3 i+2} s_{3 i-1,3 i+2} s_{3 i, 3 i+3} \mu_{3 i+1} \mu_{3 i-1} \mu_{3 i+3} \mu_{3 i+1} .
$$

Note that

$$
\stackrel{i}{\mathrm{R}^{-1}}=s_{3 i, 3 i+3} s_{3 i-1,3 i+2} s_{3 i, 3 i+2} \mu_{3 i+1} \mu_{3 i+2} \mu_{3 i} \mu_{3 i+1} .
$$

The R-operators $\stackrel{i}{R}^{ \pm 1}$ leave the exchange matrix associated to Figure 2 invariant. The explicit forms of the actions on the cluster variable $\boldsymbol{x}=\left(x_{1}, x_{2}, \ldots, x_{3 n+1}\right)$ and the $y$-variable $\boldsymbol{y}=\left(y_{1}, y_{2}, \ldots, y_{3 n+1}\right)$ are

$$
\begin{aligned}
& \stackrel{i}{\mathrm{R}} \pm 1 \\
& \stackrel{i}{i})=\left(x_{1}, \ldots, x_{3 i-3}, \mathrm{R}^{ \pm 1}\left(x_{3 i-2}, \ldots, x_{3 i+4}\right), x_{3 i+5}, \ldots, x_{3 n+1}\right), \\
& \mathrm{R}^{ \pm 1}(\boldsymbol{y})=\left(y_{1}, \ldots, y_{3 i-3}, \mathrm{R}^{ \pm 1}\left(y_{3 i-2}, \ldots, y_{3 i+4}\right), y_{3 i+5}, \ldots, y_{3 n+1}\right),
\end{aligned}
$$

where $\mathrm{R}^{ \pm 1}\left(x_{1}, \ldots, x_{7}\right)$ and $\mathrm{R}^{ \pm 1}\left(y_{1}, \ldots, y_{7}\right)$ are defined in (2-12) and (2-13) respectively.

Theorem 2.3 The R-operator satisfies the braid relation, namely we have

$$
\begin{aligned}
& \stackrel{i}{i+1} \stackrel{i}{\mathrm{R}} \mathrm{R}=\stackrel{i+1}{\mathrm{R}} \mathrm{i} \stackrel{i+1}{\mathrm{R}} \quad \text { for } i=1,2, \ldots, n-2, \\
& \stackrel{i j}{\mathrm{RR}}=\stackrel{j i}{\mathrm{RR}} \quad \text { for }|i-j|>1 \text {. }
\end{aligned}
$$


Proof The second equality is trivial.

It is sufficient to check

$$
\begin{aligned}
& 121 \\
& \text { RRR }=\text { RRR }
\end{aligned}
$$

on the cluster variable $\left(x_{1}, \ldots, x_{10}\right)$ with the exchange matrix associated to Figure 2 with $n=3$. By direct computation, we can check that both actions result in the following same expressions:

$$
\begin{array}{r}
\left(x_{1}, x_{8}, \frac{x_{1} x_{2} x_{4} x_{6} x_{8}+x_{1} x_{3} x_{5} x_{7} x_{8}+x_{3} x_{4} x_{5} x_{7} x_{8}+x_{1} x_{2} x_{6} x_{7} x_{8}+x_{1} x_{2} x_{4} x_{5} x_{9}}{x_{2} x_{4} x_{5} x_{7}},\right. \\
\left.\begin{array}{r}
\frac{1}{x_{2} x_{4} x_{5} x_{7} x_{9}}\left(\begin{array}{r}
x_{1} x_{2} x_{4} x_{6} x_{7} x_{8}+x_{1} x_{3} x_{5} x_{7}^{2} x_{8}+x_{3} x_{4} x_{5} x_{7}^{2} x_{8} \\
+x_{1} x_{2} x_{6} x_{7}^{2} x_{8}+x_{1} x_{2} x_{4} x_{6} x_{8} x_{10}+x_{1} x_{3} x_{5} x_{7} x_{8} x_{10} \\
\left.+x_{3} x_{4} x_{5} x_{7} x_{8} x_{10}+x_{1} x_{2} x_{6} x_{7} x_{8} x_{10}+x_{1} x_{2} x_{4} x_{5} x_{9} x_{10}\right), \\
\frac{x_{6} x_{7} x_{8}+x_{6} x_{8} x_{10}+x_{5} x_{9} x_{10}}{x_{7} x_{9}}, \frac{x_{1} x_{3} x_{5}+x_{3} x_{4} x_{5}+x_{1} x_{2} x_{6}}{x_{2} x_{4}}, \\
\frac{1}{x_{2} x_{4} x_{6} x_{7} x_{9}}\left(\begin{array}{r}
x_{1} x_{3} x_{4} x_{6} x_{7} x_{8}+x_{3} x_{4}^{2} x_{6} x_{7} x_{8}+x_{1} x_{3} x_{4} x_{6} x_{8} x_{10} \\
+x_{3} x_{4}^{2} x_{6} x_{8} x_{10}+x_{1} x_{3} x_{4} x_{5} x_{9} x_{10}+x_{3} x_{4}^{2} x_{5} x_{9} x_{10}
\end{array}\right. \\
\left.+x_{1} x_{3} x_{5} x_{7} x_{9} x_{10}+x_{3} x_{4} x_{5} x_{7} x_{9} x_{10}+x_{1} x_{2} x_{6} x_{7} x_{9} x_{10}\right)
\end{array},\right. \\
x_{3} x_{4} x_{6} x_{7} x_{8}+x_{3} x_{4} x_{6} x_{8} x_{10}+x_{3} x_{4} x_{5} x_{9} x_{10}+x_{3} x_{5} x_{7} x_{9} x_{10}+x_{2} x_{6} x_{7} x_{9} x_{10} \\
x_{4} x_{6} x_{7} x_{9}
\end{array}\right) .
\end{array}
$$

Actions on the $y$-variables are induced from these actions. This completes the proof.

The R-operator (2-9) is not new. In [18], Kashaev constructs a solution of the YangBaxter equation from the quantum dilogarithm function based on a relationship with Teichmüller theory. An operator which has a similar action on the $y$-variable (2-13) was used in studies of lamination [5]. In our case, the braiding denotes an exchange of the punctures on the disk. Also an operator which has a tropicalized action of cluster variable (2-12) was given in [4]. See Fock and Goncharov [6] for applications of Teichmüller coordinates to laminations.

\section{Hyperbolic geometry}

\subsection{Ideal tetrahedron}

A building block of hyperbolic 3-manifold is an ideal tetrahedron whose vertices are on the boundary of a hyperbolic 3-space; see W Thurston [25]. An ideal hyperbolic 
tetrahedron $\triangle$ is parameterized with cross ratio $z \in \mathbb{C}$ of its four vertices, and the volume of $\triangle$ is given by the Bloch-Wigner function,

$$
D(z)=\Im \operatorname{Li}_{2}(z)+\arg (1-z) \log |z| .
$$

As depicted in Figure 3, opposite edges have the same dihedral angles. Therein we have used the notation

$$
z^{\prime}=1-\frac{1}{z}, \quad z^{\prime \prime}=\frac{1}{1-z}
$$
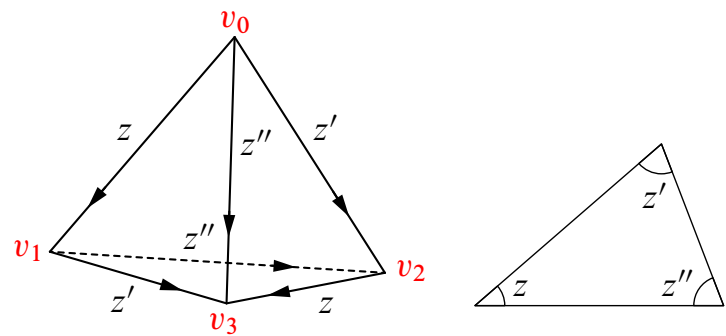

Figure 3: An oriented ideal tetrahedron (left) and a triangle as intersection with horosphere (right)

In case a set of ideal tetrahedra $\left\{\triangle_{v}\right\}$ is glued faces together to a hyperbolic manifold $M=\bigcup_{v} \triangle_{v}$, the volume of $M$ is given by

$$
\operatorname{Vol}(M)=\sum_{v} D\left(z_{v}\right)
$$

A complexification of $\operatorname{Vol}(M)$, known as complex volume, is defined via the ChernSimons invariant $\operatorname{CS}(M)$. We have [23]

$$
\mathrm{i}(\operatorname{Vol}(M)+\mathrm{i} C S(M))=\sum_{v} \operatorname{sgn}\left(\triangle_{v}\right) \mathrm{L}\left(\left[z_{v} ; p_{\nu}, q_{v}\right]\right),
$$

where $\left[z_{v} ; p_{v}, q_{v}\right]$ is an element of the extended Bloch group with integers $p_{v}$ and $q_{v}$, and $\operatorname{sgn}\left(\triangle_{v}\right)$ is +1 (resp. -1 ) when the vertex ordering of $\triangle_{v}$ is same (resp. inverse) with Figure 3. We have used the extended Rogers dilogarithm function

$$
\mathrm{L}([z ; p, q])=\mathrm{Li}_{2}(z)+\frac{1}{2} \log z \log (1-z)+\frac{\pi \mathrm{i}}{2}(q \log z+p \log (1-z))-\frac{\pi^{2}}{6} .
$$

A method to compute $p_{v}$ and $q_{v}$ was proposed in [29]. For an oriented ideal tetrahedron of modulus $z$ in Figure 3, let $c_{a b}$ be complex parameters on the edge connecting vertices $v_{a}$ and $v_{b}$. Assume that they fulfill

$$
\frac{c_{03} c_{12}}{c_{02} c_{13}}= \pm z, \quad \frac{c_{01} c_{23}}{c_{03} c_{12}}= \pm\left(1-\frac{1}{z}\right), \quad \frac{c_{02} c_{13}}{c_{01} c_{23}}= \pm \frac{1}{1-z}
$$


Note that, in gluing tetrahedra together, identical edges have the same complex parameter. Then $[z ; p, q]$, integers $p$ and $q$ for modulus $z$, is given by

$$
\begin{aligned}
\log z+p \pi \mathrm{i} & =\log c_{03}+\log c_{12}-\log c_{02}-\log c_{13}, \\
-\log (1-z)+q \pi \mathrm{i} & =\log c_{02}+\log c_{13}-\log c_{01}-\log c_{23} .
\end{aligned}
$$

Here and hereafter we mean the principal branch in the logarithm. In [29] these edge parameters $c_{a b}$ are read from a developing map.

\subsection{Octahedron}

In our previous paper [13], we demonstrated that the cluster mutation can be regarded as an attachment of ideal tetrahedron to triangulated surface (see also Nagao, Terashima and Yamazaki [21]). Furthermore we claimed that the cluster variable $\boldsymbol{x}$ corresponds to Zickert's complex parameters $c_{a b}$ on edges (see [13, Section 2.3] for details). Roughly speaking, this is due to the fact that all mutations used in $\mathrm{R}^{ \pm 1}(\boldsymbol{x})$ have the form of the Ptolemy relation, $a c+b d=e f$, which is the same as (3-6).
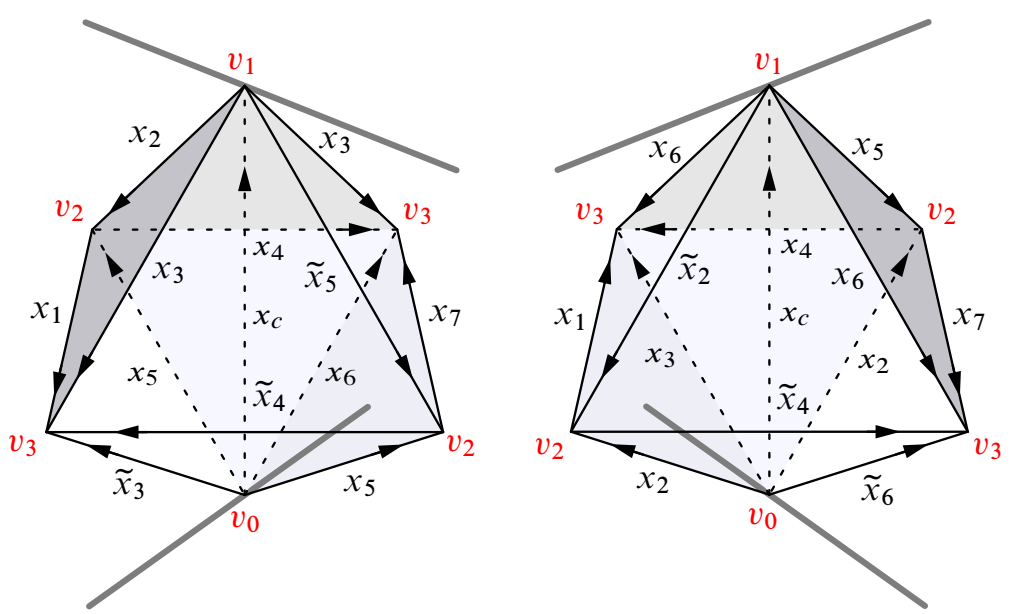

Figure 4: Octahedron for $\stackrel{1}{\mathrm{R}}$ (left) and $\stackrel{1}{\mathrm{R}}^{-1}$ (right)

For brevity, we study the case

$$
\tilde{\boldsymbol{x}}=\stackrel{1}{\mathrm{R}}^{ \pm 1}(\boldsymbol{x}) .
$$

Based on the observation in [13], we see that the R-operator (2-9) is realized as an octahedron in Figure 4, which is composed of four tetrahedra $\left\{\triangle_{N}, \triangle_{S}, \triangle_{W}, \triangle_{E}\right\}$. See Figure 5 for a top view. The four tetrahedra originate from four mutations in the $\mathrm{R}^{ \pm 1}$-operator, (2-9) and (2-10); $\mu_{2}$ and $\mu_{6}$ in (2-9) respectively correspond to $\triangle_{W}$ 
and $\triangle_{E}$, and two $\mu_{4}$ are for $\triangle_{N}$ and $\Delta_{S}$. The cluster variables $x_{k}$ and $\tilde{x}_{k}$ are assigned to edges of the octahedra, and we have used

$$
x_{c}=\frac{x_{2} x_{6}+x_{3} x_{5}}{x_{4}} .
$$

Note that we have fixed vertex ordering for our convention, and that edges with the same complex parameters (eg two pairs of edges $v_{0}-v_{2}, v_{1}-v_{3}$ ) are identical.

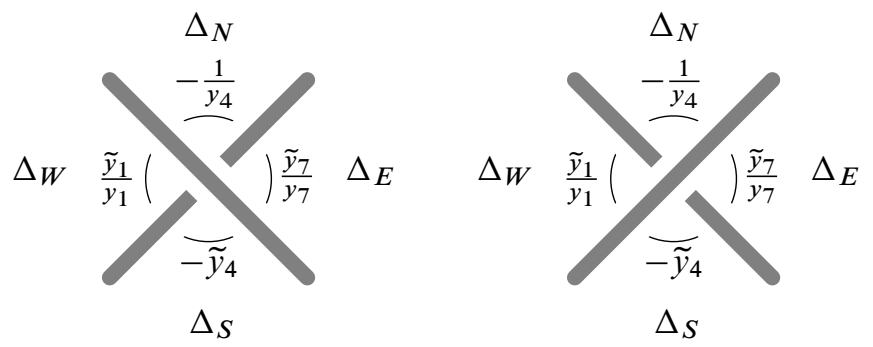

Figure 5: Dihedral angle at crossings, $\stackrel{1}{\mathrm{R}}$ (left) and $\stackrel{1}{\mathrm{R}}^{-1}$ (right)

As the R-operator satisfies the braid relation (Theorem 2.3), we can interpret that each octahedron is assigned to every crossing of knot diagram as in Figure 5. This is similar to a fact of Thurston [24] that octahedron was assigned to the Kashaev R-matrix [16] (see also the first author [11], Cho, H Kim, Hyuk and S Kim [1], Cho, J Murakami and Yokota [3] and Yokota [27]). Note that another expression (2-14) of the same $\mathrm{R}$-operator corresponds to a decomposition of octahedron into five tetrahedra, which was used in studies of the colored Jones R-matrix at root of unity; see D Thurston [24] and Cho and J Murakami [2].

\begin{tabular}{|c|c|c|c|c|c|c|c|}
\hline \multirow[b]{2}{*}{$\triangle$} & \multirow[b]{2}{*}{ Volume } & \multicolumn{3}{|c|}{$\begin{array}{l}1 \\
\mathrm{R}\end{array}$} & \multicolumn{3}{|c|}{$\mathrm{R}^{-1}$} \\
\hline & & $\operatorname{sgn}(\triangle)$ & $z_{\triangle}$ & $\frac{1}{1-z_{\triangle}}$ & $\operatorname{sgn}(\triangle)$ & $z_{\triangle}$ & $\frac{1}{1-z_{\Delta}}$ \\
\hline$\triangle_{N}$ & $D\left(-\frac{1}{y_{4}}\right)$ & - & $-\frac{x_{2} x_{6}}{x_{3} x_{5}}$ & $\frac{x_{3} x_{5}}{x_{4} x_{c}}$ & + & $-\frac{x_{3} x_{5}}{x_{2} x_{6}}$ & $\frac{x_{2} x_{6}}{x_{4} x_{c}}$ \\
\hline$\triangle_{S}$ & $D\left(-\tilde{y}_{4}\right)$ & - & $-\frac{\tilde{x}_{3} \tilde{x}_{5}}{x_{3} x_{5}}$ & $\frac{x_{3} x_{5}}{\tilde{x}_{4} x_{c}}$ & + & $-\frac{\tilde{x}_{2} \tilde{x}_{6}}{x_{2} x_{6}}$ & $\frac{x_{2} x_{6}}{x_{c} \tilde{x}_{4}}$ \\
\hline$\triangle_{W}$ & $D\left(\frac{\tilde{y}_{1}}{y_{1}}\right)$ & + & $\frac{x_{2} \tilde{x}_{3}}{x_{3} x_{5}}$ & $-\frac{x_{3} x_{5}}{x_{1} x_{c}}$ & - & $\frac{\tilde{x}_{2} x_{3}}{x_{2} x_{6}}$ & $-\frac{x_{2} x_{6}}{x_{1} x_{c}}$ \\
\hline$\triangle_{E}$ & $D\left(\frac{\tilde{y}_{7}}{y_{7}}\right)$ & + & $\frac{\tilde{x}_{5} x_{6}}{x_{3} x_{5}}$ & $-\frac{x_{3} x_{5}}{x_{c} x_{7}}$ & - & $\frac{x_{5} \tilde{x}_{6}}{x_{2} x_{6}}$ & $-\frac{x_{2} x_{6}}{x_{c} x_{7}}$ \\
\hline
\end{tabular}

Table 1: Moduli of four tetrahedra assigned to operators $\mathrm{R}^{1}$ and $\mathrm{R}^{-1}$ : sign "+" (resp. "-") means that vertex ordering of tetrahedron is same (resp. inverse) with Figure 3. 
Taking into account of the vertex ordering of tetrahedra, we can determine moduli of each tetrahedron from (3-6) as in Table 1. From these results, we define dilogarithm functions for every crossing by

$$
\mathrm{L}\left(\left[\mathrm{R}^{ \pm}\right] ; \boldsymbol{x}\right)=\sum_{t \in\{N, S, W, E\}} \operatorname{sgn}\left(\triangle_{t}\right) \mathrm{L}\left(\left[z_{\triangle_{t}} ; p_{\triangle_{t}}, q_{\triangle_{t}}\right]\right) .
$$

Here we have used the extended Rogers dilogarithm (3-5), and integers $p_{\triangle_{t}}$ and $q_{\triangle_{t}}$ are given from (3-7) by use of Table 1 .

For instance, $p_{\triangle_{E}}$ and $q_{\triangle_{E}}$ in the operator $\mathrm{R}$ are given as

$$
\begin{aligned}
& p_{\triangle_{E}} \pi \mathrm{i}=-\log \left(\frac{\tilde{x}_{5} x_{6}}{x_{3} x_{5}}\right)+\log \left(\tilde{x}_{5}\right)+\log \left(x_{6}\right)-\log \left(x_{3}\right)-\log \left(x_{5}\right), \\
& q_{\triangle_{E}} \pi \mathrm{i}=-\log \left(-\frac{x_{3} x_{5}}{x_{c} x_{7}}\right)+\log \left(x_{3}\right)+\log \left(x_{5}\right)-\log \left(x_{c}\right)-\log \left(x_{7}\right) .
\end{aligned}
$$

It should be remarked that, to identify the R-operator with a hyperbolic octahedron, we need a consistency condition around a central edge labeled by $x_{c}$ in Figure 4. This condition is automatically satisfied by

$$
y_{1} y_{4} y_{7}=\tilde{y}_{1} \tilde{y}_{4} \tilde{y}_{7},
$$

where $\tilde{\boldsymbol{y}}=\stackrel{1}{\mathrm{R}}^{ \pm 1}(\boldsymbol{y})$; see (2-18). In Figure 5 denoted are dihedral angles around central axis assigned to each crossing.

We can interpret the $i^{\text {th }}$ braiding operator $\stackrel{i}{\mathrm{R}}^{ \pm 1}$ in (2-15) in the same manner. As we have the cluster mutation

$$
\tilde{\boldsymbol{x}}=\stackrel{i}{\mathrm{R}} \pm 1(\boldsymbol{x})
$$

as in (2-17), the edge parameters $\left(x_{1}, x_{2}, \ldots, x_{7}\right)$ and $\left(\tilde{x}_{1}, \tilde{x}_{2}, \ldots, \tilde{x}_{7}\right)$ in Figure 4 are replaced respectively by $\left(x_{3 i-2}, x_{3 i-1}, \ldots, x_{3 i+4}\right)$ and $\left(\tilde{x}_{3 i-2}, \tilde{x}_{3 i-1}, \ldots, \tilde{x}_{3 i+4}\right)$. The moduli of the tetrahedra in Table 1 should be replaced correspondingly, and as a result we have the dilogarithm function as in (3-9) by replacing $x_{a}$ with $x_{3 i+a-3}$.

\subsection{Braid group presentation and gluing conditions}

Our main claim is the following.

Theorem 3.1 Let a knot $K$ have a braid group presentation $\sigma_{k_{1}}^{\varepsilon_{1}} \sigma_{k_{2}}^{\varepsilon_{2}} \cdots \sigma_{k_{m}}^{\varepsilon_{m}}$, where $\varepsilon_{j}= \pm 1$ and

$$
\begin{aligned}
& \mathcal{B}_{n}=\left\langle\sigma_{1}, \sigma_{2}, \ldots, \sigma_{n-1}\right| \sigma_{i} \sigma_{j}=\sigma_{j} \sigma_{i} \text { for }|i-j|>1, \\
&\left.\sigma_{i} \sigma_{i+1} \sigma_{i}=\sigma_{i+1} \sigma_{i} \sigma_{i+1} \text { for } i=1,2, \ldots, n-2\right\rangle .
\end{aligned}
$$

We define a cluster pattern for $\boldsymbol{x}[j]=\left(x[j]_{1}, \ldots, x[j]_{3 n+1}\right)$ by 


$$
\boldsymbol{x}[1] \stackrel{k_{1} \varepsilon_{1}}{\longrightarrow} \boldsymbol{x}[2] \stackrel{k_{2} \varepsilon_{2}}{\longrightarrow} \cdots \stackrel{k_{\mathrm{R}}{ }^{k_{m}}}{\longrightarrow} \boldsymbol{x}[m+1],
$$

with the exchange matrix associated to Figure 2. We assume that the initial cluster variable $\boldsymbol{x}[1]$ satisfies

$$
\boldsymbol{x}[1]=\boldsymbol{x}[m+1] .
$$

Then the $y$-variables, $y[k]_{i} \in \mathbb{C}$, induced from the cluster pattern fulfill algebraic equations for shape parameters of ideal tetrahedra in the triangulation of $S^{3} \backslash(K \cup$ 2-points).

We note that the periodicity (3-11) denotes a closure of the braid, and that the 2-points are $v_{2}$ and $v_{3}$ in Figure 4.

In the above theorem, we do not assume that a knot $K$ is hyperbolic. We study a triangulation induced from a braid group presentation. This situation is the same with the volume conjecture [17], which suggests an intimate relationship between a complex volume of $S^{3} \backslash K$ and the Kashaev invariant for $K$ defined from a quantum $R$-matrix.

Our triangulation is a standard one used in SnapPy (see Weeks [26]), and the NeumannZagier potential function was constructed in [3] from such triangulation. See also A Inoue and Kabaya [15], where complex volume is studied from the same triangulation by use of quandle. So it is natural to expect that for hyperbolic knot $K$ there exists a geometric solution of (3-11), where the neighbors of additional two points cancel and we endow a complete hyperbolic structure for $S^{3} \backslash K$. We show in the next section numerical results for some knots, and we discuss how the cancellation of two balls occurs (see Proposition 4.1). Unfortunately, at this stage, we do not know how to extract generally such a preferable solution from (3-11). Due to the fact that the geometric content of each octahedron is identified as in Table 1, we obtain complex volume as follows if we assume the existence of a geometric solution.

Conjecture 3.2 There exists an algebraic solution of (3-11) such that the complex volume of $K$ is given by

$$
\mathrm{i}\left(\operatorname{Vol}\left(S^{3} \backslash K\right)+\mathrm{i} \operatorname{CS}\left(S^{3} \backslash K\right)\right)=\sum_{j=1}^{m} \mathrm{~L}\left(\left[\mathrm{R}^{k_{j}}\right] ; \boldsymbol{x}[j]\right) .
$$

The definition of the dilogarithm function $\mathrm{L}\left(\left[\mathrm{R}^{\varepsilon_{j}}\right] ; \boldsymbol{x}[j]\right)$ can be found in (3-9) and the end of the last subsection.

Proof of Theorem 3.1 We need to check consistency conditions and completeness conditions as $[11 ; 1]$. We have already seen that a consistency condition around a 
central axis of octahedra is fulfilled. We shall check other cases. First we study a cluster pattern

$$
\boldsymbol{x}[1] \stackrel{\stackrel{\mathrm{R}}{\rightarrow}}{\rightarrow} \boldsymbol{x}[2] \stackrel{2}{\stackrel{\mathrm{R}}{\rightarrow}} \boldsymbol{x}[3] .
$$

For each crossing we assign octahedra as in Figure 6. Therein colored faces are glued together so that identical edges have the same complex parameters. Note that (2-12) implies $x[2]_{6}=x[1]_{3}$ and $x[1]_{7}=x[2]_{7}$, and that

$$
x[1]_{c}=\frac{x[1]_{2} x[1]_{6}+x[1]_{3} x[1]_{5}}{x[1]_{4}}, \quad x[2]_{c}=\frac{x[2]_{5} x[2]_{9}+x[2]_{6} x[2]_{8}}{x[2]_{7}} .
$$

Consistency condition around edge labeled by complex parameter $x[2]_{5}$ is checked as

$$
\frac{1}{1-\frac{y[2]_{7}}{y[1]_{7}}} \cdot\left(1+\frac{1}{y[2]_{4}}\right) \cdot\left(1+y[2]_{7}\right) \cdot \frac{1}{1-\frac{y[3]_{4}}{y[2]_{4}}}=1 .
$$
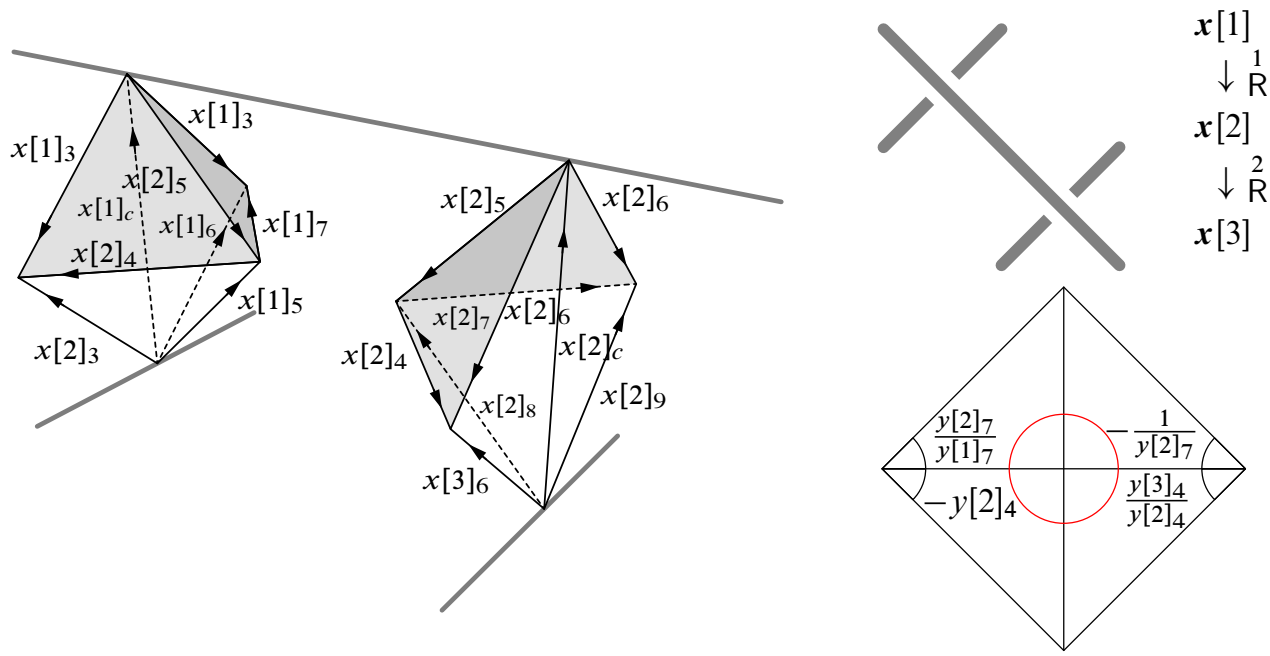

Figure 6: Gluing of octahedra (left) assigned to crossing (right top), and a developing map (right bottom): consistency is read from the red circle.

The same goes for a case of a cluster pattern

$$
x[1] \stackrel{\mathrm{R}^{-1}}{\longrightarrow} x[2] \stackrel{\mathrm{R}^{-1}}{\longrightarrow} x[3] .
$$

We have octahedra as in Figure 7, and we can check a consistency condition in the developing map as

$$
\left(1-\frac{y[1]_{7}}{y[2]_{7}}\right) \cdot \frac{1}{1+y[2]_{4}} \cdot\left(1-\frac{y[2]_{4}}{y[3]_{4}}\right) \cdot \frac{1}{1+\frac{1}{y[2]_{7}}}=1 .
$$



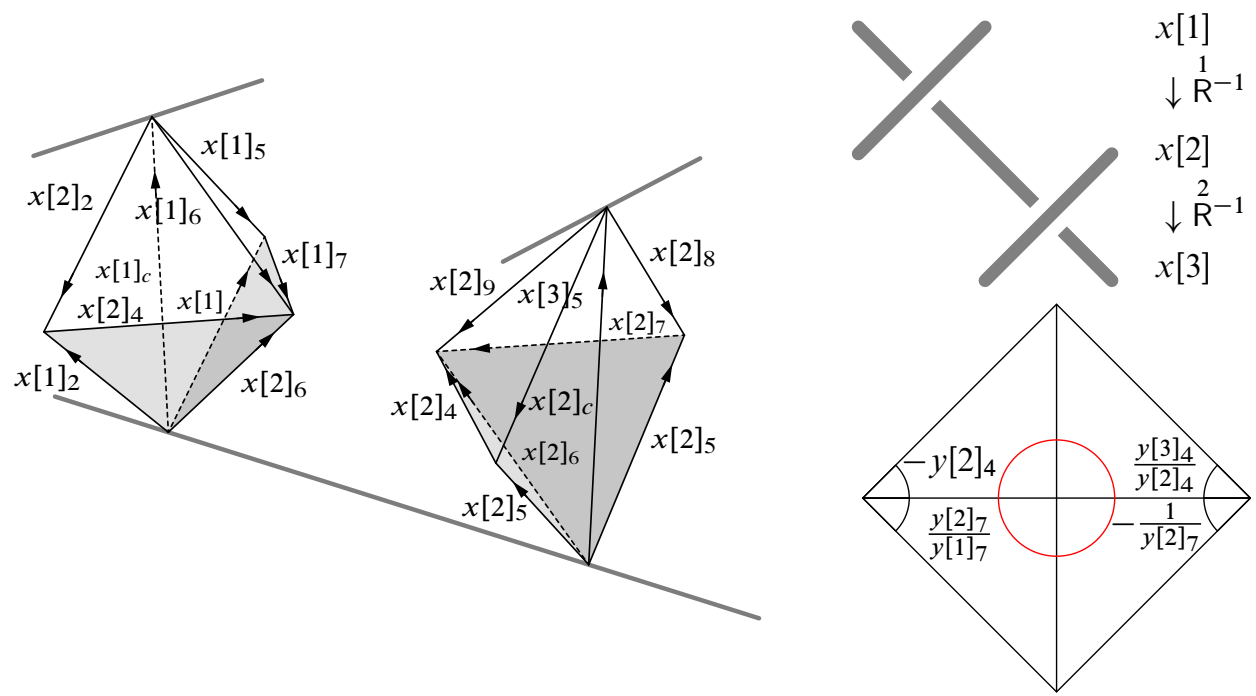

Figure 7: Gluing of octahedra (left) assigned to crossing (right top), and a developing map (right bottom): consistency is read from the red circle.
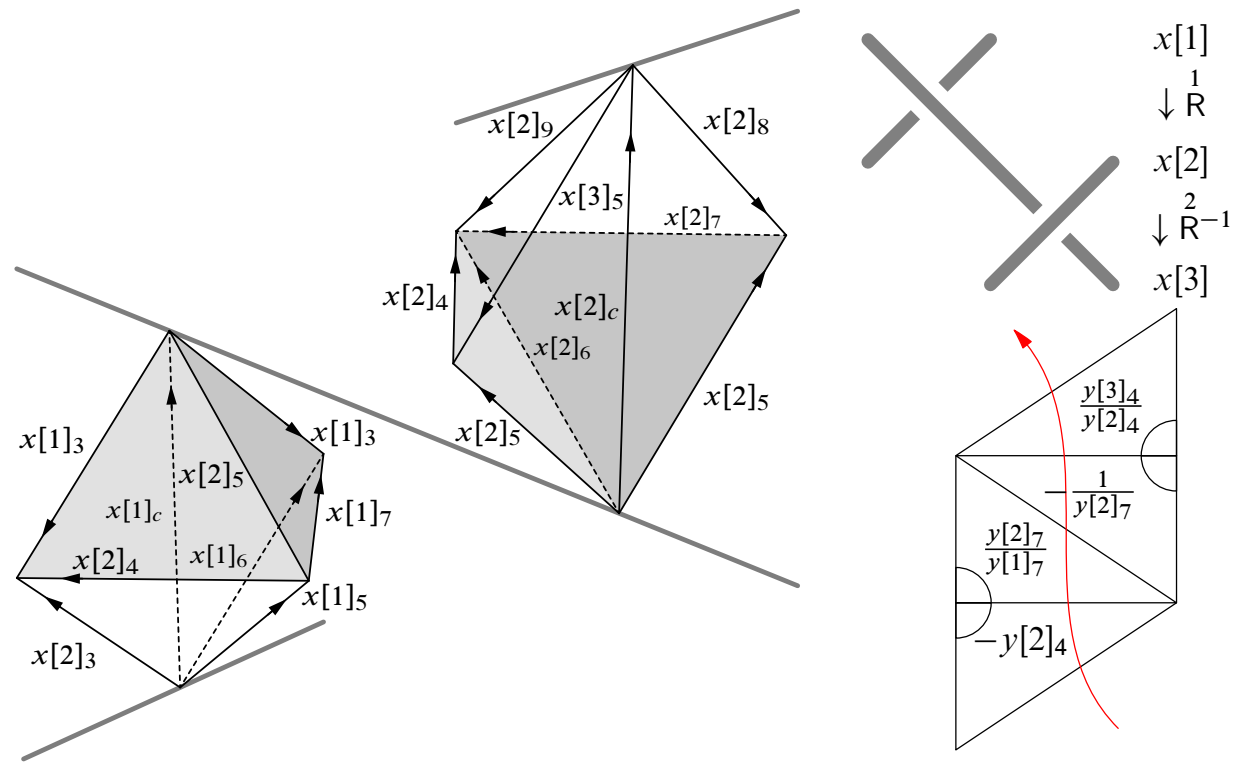

Figure 8: Gluing of octahedra (left) assigned to crossing (right top), and a developing map (right bottom): completeness is read from the red curve.

A completeness condition follows from alternating crossings. In the case that the cluster pattern is given by

$$
x[1] \stackrel{1}{\rightarrow} x[2] \stackrel{\mathrm{R}^{-1}}{\longrightarrow} x[3]
$$


octahedra are attached to each crossing as in Figure 8. See that identical edges have the same complex parameters $x[2]_{6}=x[1]_{3}$ and $x[1]_{7}=x[2]_{7}$ due to (2-12). Then we can check the completeness condition as

$$
\frac{1+\frac{1}{y[2]_{4}}}{1-\frac{y[2]_{7}}{y[1]_{7}}} \cdot \frac{1+\frac{1}{y[2]_{7}}}{1-\frac{y[2]_{4}}{y[3]_{4}}}=y[1]_{2} y[1]_{3}=1
$$

Here the last equality follows from (2-4).

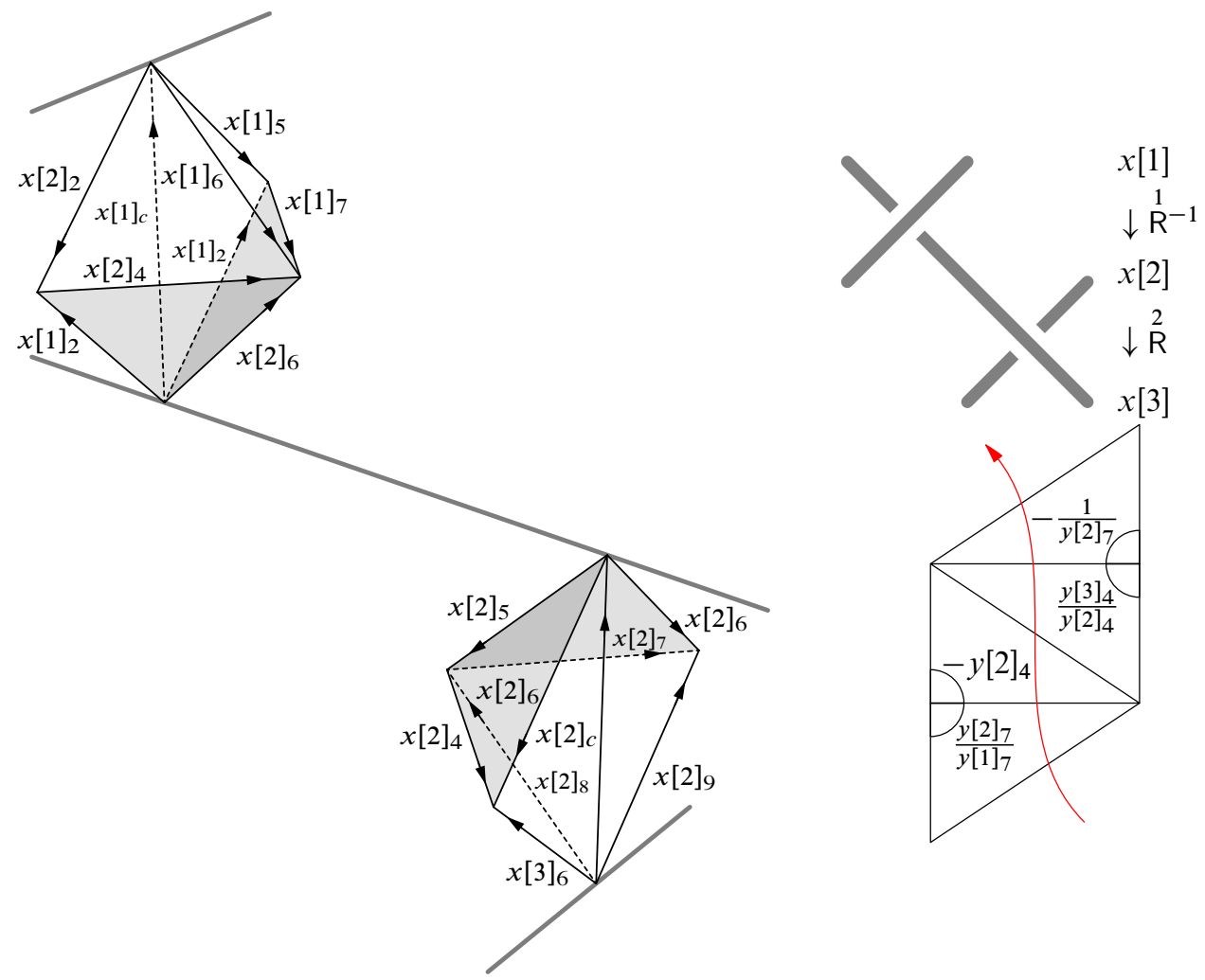

Figure 9: Gluing of octahedra (left) assigned to crossing (right top) and a developing map (right bottom)

Figure 9 corresponds to the cluster pattern

$$
x[1] \stackrel{\mathrm{R}^{-1}}{\longrightarrow} x[2] \stackrel{\stackrel{2}{\rightarrow}}{\rightarrow} x[3] .
$$


By use of (2-4), we have a completeness condition

$$
\frac{1-\frac{y[1]_{7}}{y[2]_{7}}}{1+y[2]_{4}} \cdot \frac{1-\frac{y[3]_{4}}{y[2]_{4}}}{1+y[2]_{7}}=y[1]_{2} y[1]_{3}=1 .
$$

Other cases can be checked in a similar manner, and the claim follows.

We note that in the above proof the completeness condition is

$$
y[1]_{3 i-1} y[1]_{3 i}=1 \text { for } i=1,2, \ldots, n,
$$

which follows from the definition of the $y$-variables (2-4).

\section{Examples}

\subsection{Figure-eight knot $\mathbf{4}_{1}$}

We study the figure-eight knot whose braid group presentation is $\sigma_{1} \sigma_{2}^{-1} \sigma_{1} \sigma_{2}^{-1}$. The cluster pattern for $4_{1}$ is thus

$$
x[1] \stackrel{1}{\rightarrow} x[2] \stackrel{\mathrm{R}^{-1}}{\longrightarrow} x[3] \stackrel{\stackrel{\mathrm{R}}{\rightarrow}}{\rightarrow} x[4] \stackrel{\stackrel{2}{\mathrm{R}}^{-1}}{\longrightarrow} x[5] .
$$

We can check that $\boldsymbol{x}[1]=\boldsymbol{x}[5]$ is fulfilled by, for example,

$$
\boldsymbol{x}[1]=\left(x_{1}, x_{2}, x_{2}, 1, x_{1} x_{2}, x_{1}^{2} x_{2}, x_{1},-x_{2},-x_{2}, 1\right),
$$

where $\left(x_{1}, x_{2}\right)=\left(\mathrm{e}^{2 \pi \mathrm{i} / 3}, 0\right)$. To compute the complex volume of $4_{1}$, we set $\left(x_{1}, x_{2}\right)=$ $\left(\mathrm{e}^{2 \pi \mathrm{i} / 3}+\delta, \delta\right)$ with $\delta \in \mathbb{R}_{>0}$, and take a limit $\delta \rightarrow 0$. We have checked numerically that (3-12) gives $\mathrm{i} \cdot 2 D\left(\mathrm{e}^{\pi \mathrm{i} / 3}\right)=\mathrm{i} \cdot 2.02988 \ldots$ as desired [25].

\subsection{Trefoil knot $3_{1}$}

The next example is the trefoil $3_{1}$, which is not hyperbolic. The braid group presentation for $3_{1}$ is $\sigma_{1}^{3}$, and its cluster pattern is

$$
\boldsymbol{x}[1] \stackrel{\mathrm{R}^{\prime}}{\rightarrow} \boldsymbol{x}[2] \stackrel{\stackrel{\mathrm{R}}{\rightarrow}}{\rightarrow} \boldsymbol{x}[3] \stackrel{\stackrel{\mathrm{R}}{\rightarrow}}{\rightarrow} \boldsymbol{x}[4] .
$$

We solve $\boldsymbol{x}[1]=\boldsymbol{x}[4]$ by choosing an initial cluster variable as

$$
\boldsymbol{x}[1]=\left(x_{1}, x_{2}, x_{2}, 1, x_{1} x_{2}, x_{1}^{2} x_{2}, 1\right)
$$

and get $x_{1}=-(1+\mathrm{i}) / 2$ in the limit $x_{2} \rightarrow 0$. We check numerically that (3-12) gives $-8.22467 \ldots \simeq-\frac{5}{6} \pi^{2}$. It agrees with the Chern-Simons invariant of $3_{1}$, which is also given from asymptotic limit of the Kashaev invariant; see Kashaev and Tirkkonen [19], Zagier [28] and the first author and Kirillov [14]. 


\subsection{Interpretation of initial cluster variables}

In the above examples, we have singular solutions such as

$$
\frac{x[1]_{2}}{x[1]_{1}}, \frac{x[1]_{3}}{x[1]_{4}} \rightarrow 0 .
$$

This condition for initial cluster variables denotes that a cancellation of the two additional balls occurs by connecting to the tubular neighbor of the knot $K$ as explained in [26] (see also [3]).

Proposition 4.1 In the setting of Theorem 3.1, when we set an initial cluster $x-$ variable as (4-2), we get a canonical triangulation of $S^{3} \backslash K$.

We note that such cancellation can occur under other choices of initial cluster variables.

Proof of Proposition 4.1 We need to connect two balls at $v_{2}$ and $v_{3}$ (see Figure 4) to the tubular neighbor of the knot $K$ to get a triangulation of $S^{3} \backslash K$. For this purpose, we introduce a triangular pillow with a predrilled tube as in Figure 10. The pillow is constructed from two hyperbolic tetrahedra as in Figure 10, and we see that there exists a drilled tube connecting two vertices (see [26]). By use of other hyperbolic tetrahedra whose vertex orderings are opposite to those in Figure 10, we have another type of a triangular pillow as in Figure 11.
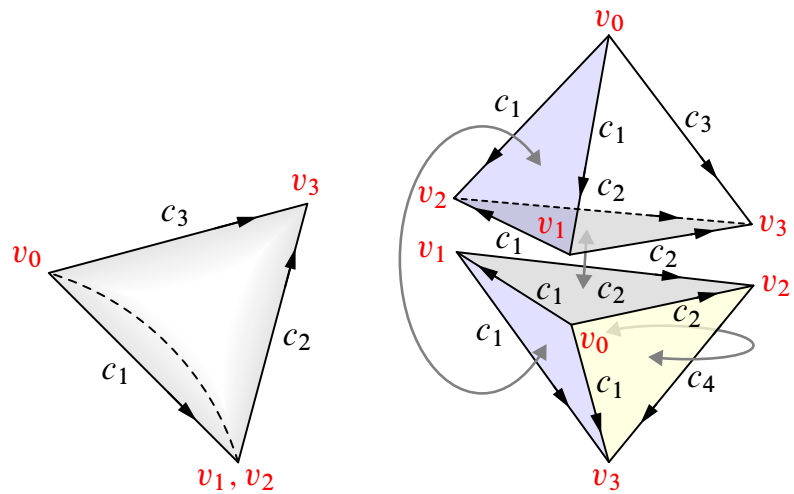

Figure 10: A pillow with a predrilled tube (left) is constructed from two ideal tetrahedra (right) by gluing colored faces together. A dashed curve denotes a tube connecting two vertices. Here $c_{a}$ is a edge parameter.

In both Figures 10 and 11 , we assign edge parameters $c_{a}$ for each edge. Shape parameters of tetrahedra are given by (3-6), and we get

$$
\frac{c_{3}}{c_{2}}=0
$$


Because of their opposite vertex orderings, a sum of the extended Rogers dilogarithm functions (3-5) for two pillows vanishes.
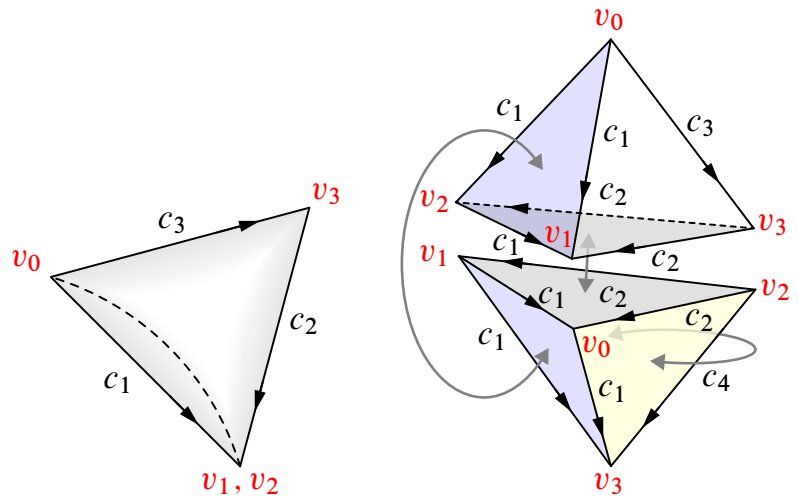

Figure 11: Another pillow with a predrilled tube (left) is given from two hyperbolic tetrahedra (right).

We insert and glue the pillow in Figure 10 (resp. Figure 11) to the triangular surface $x_{2} x_{3} x_{4}$ in $\Delta_{N}$ (resp. $x_{1} x_{2} x_{3}$ in $\Delta_{W}$ ) in the octahedron assigned to the first crossing R. Predrilled tubes of the pillows connect both vertices $v_{2}$ and $v_{3}$ to $v_{1}$ in Figure 4. To conclude, we obtain a valid triangulation of $S^{3} \backslash K$. Since identical edges have the same edge parameters, condition (4-3) gives $x[1]_{2} / x[1]_{1}=x[1]_{3} / x[1]_{4}=0$.

Acknowledgments The authors would like to thank Jun Murakami for stimulating discussions and for comments on the manuscript. Thanks are also to Rinat Kashaev for bringing [5] to our attention. The authors are grateful for anonymous referee's helpful comments. The work of KH is supported in part by JSPS KAKENHI grant number 23340115,24654041 . The work of RI is partially supported by JSPS KAKENHI grant number 22740111.

\section{References}

[1] J Cho, H Kim, S Kim, Optimistic limits of Kashaev invariants and complex volumes of hyperbolic links, J. Knot Theory Ramifications 23 (2014) 1450049 MR3268985

[2] J Cho, J Murakami, The complex volumes of twist knots via colored Jones polynomials, J. Knot Theory Ramifications 19 (2010) 1401-1421 MR2746194

[3] J Cho, J Murakami, Y Yokota, The complex volumes of twist knots, Proc. Amer. Math. Soc. 137 (2009) 3533-3541 MR2515423

[4] P Dehornoy, I Dynnikov, D Rolfsen, B Wiest, Ordering braids, Math. Surveys and Monographs 148, Amer. Math. Soc. (2008) MR2463428 
[5] I A Dynnikov, On a Yang-Baxter mapping and the Dehornoy ordering, Uspekhi Mat. Nauk 57 (2002) 151-152 MR1918864 In Russian; translated in Russ. Math. Surveys 57 (2012) 592-594

[6] V V Fock, A B Goncharov, Dual Teichmüller and lamination spaces, from: "Handbook of Teichmüller theory, Volume I", (A Papadopoulos, editor), IRMA Lect. Math. Theor. Phys. 11, Eur. Math. Soc., Zürich (2007) 647-684 MR2349682

[7] V V Fock, A B Goncharov, Cluster ensembles, quantization and the dilogarithm, Ann. Sci. Éc. Norm. Supér. 42 (2009) 865-930 MR2567745

[8] S Fomin, M Shapiro, D Thurston, Cluster algebras and triangulated surfaces, I: Cluster complexes, Acta Math. 201 (2008) 83-146 MR2448067

[9] S Fomin, A Zelevinsky, Cluster algebras, I: Foundations, J. Amer. Math. Soc. 15 (2002) 497-529 MR1887642

[10] S Fomin, A Zelevinsky, Cluster algebras, IV: Coefficients, Compos. Math. 143 (2007) 112-164 MR2295199

[11] K Hikami, Hyperbolic structure arising from a knot invariant, Internat. J. Modern Phys. A 16 (2001) 3309-3333 MR1848458

[12] K Hikami, R Inoue, Braiding operator via quantum cluster algebra, J. Phys. A 47 (2014) 474006, 21 MR3279997

[13] K Hikami, R Inoue, Cluster algebra and complex volume of once-punctured torus bundles and 2-bridge links, J. Knot Theory Ramifications 23 (2014) 1450006, 33 MR3190130

[14] K Hikami, A N Kirillov, Torus knot and minimal model, Phys. Lett. B 575 (2003) 343-348 MR2044711

[15] A Inoue, Y Kabaya, Quandle homology and complex volume, Geom. Dedicata 171 (2014) 265-292 MR3226796

[16] R M Kashaev, A link invariant from quantum dilogarithm, Modern Phys. Lett. A 10 (1995) 1409-1418 MR1341338

[17] R M Kashaev, The hyperbolic volume of knots from the quantum dilogarithm, Lett. Math. Phys. 39 (1997) 269-275 MR1434238

[18] R M Kashaev, On the spectrum of Dehn twists in quantum Teichmüller theory, from: "Physics and combinatorics", (A N Kirillov, N Liskova, editors), World Sci. Publ., Singapore (2001) 63-81 MR1872252

[19] R M Kashaev, O Tirkkonen, A proof of the volume conjecture on torus knots, Zap. Nauchn. Sem. S.-Peterburg. Otdel. Mat. Inst. Steklov. (POMI) 269 (2000) 262-268, 370 MR1805865

[20] H Murakami, J Murakami, The colored Jones polynomials and the simplicial volume of a knot, Acta Math. 186 (2001) 85-104 MR1828373 
[21] K Nagao, Y Terashima, M Yamazaki, Hyperbolic 3-manifolds and cluster algebras arXiv:1112.3106

[22] T Nakanishi, Periodicities in cluster algebras and dilogarithm identities, from: "Representations of algebras and related topics”, (A Skowroński, K Yamagata, editors), Eur. Math. Soc., Zürich (2011) 407-443 MR2931902

[23] W D Neumann, Extended Bloch group and the Cheeger-Chern-Simons class, Geom. Topol. 8 (2004) 413-474 MR2033484

[24] D Thurston, Hyperbolic volume and the Jones polynomial (1999) lecture notes from the summer school "Invariants de nœuds et de variétés de dimension 3" at Institut Fourier, Grenoble

[25] W P Thurston, The geometry and topology of three-manifolds, Princeton Univ. Math. Dept. Lecture Notes (1979) Available at http://msri.org/publications/books/ gt $3 \mathrm{~m} /$

[26] J Weeks, Computation of hyperbolic structures in knot theory, from: "Handbook of knot theory", (W Menasco, M Thistlethwaite, editors), Elsevier, Amsterdam (2005) 461-480 MR2179268

[27] Y Yokota, On the complex volume of hyperbolic knots, J. Knot Theory Ramifications 20 (2011) 955-976 MR2819177

[28] D Zagier, Vassiliev invariants and a strange identity related to the Dedekind etafunction, Topology 40 (2001) 945-960 MR1860536

[29] C K Zickert, The volume and Chern-Simons invariant of a representation, Duke Math. J. 150 (2009) 489-532 MR2582103

Faculty of Mathematics, Kyushu University

Fukuoka 819-0395, Japan

Department of Mathematics and Informatics, Faculty of Science, Chiba University Chiba 263-8522, Japan

khikami@gmail.com, reiiy@math.s.chiba-u.ac.jp

Received: 28 April $2014 \quad$ Revised: 6 November 2014 